\title{
Chemical Entropy Generation and MHD Effects on the Unsteady Heat and Fluid Flow through a Porous Medium
}

\author{
Gamal M. Abdel-Rahman Rashed \\ Department of Mathematics, Faculty of Science, Benha University, Benha 13518, Egypt \\ Correspondence should be addressed to Gamal M. Abdel-Rahman Rashed; gamalm60@yahoo.com
}

Received 25 November 2015; Accepted 27 December 2015

Academic Editor: Oluwole D. Makinde

Copyright ( 2016 Gamal M. Abdel-Rahman Rashed. This is an open access article distributed under the Creative Commons Attribution License, which permits unrestricted use, distribution, and reproduction in any medium, provided the original work is properly cited.

\begin{abstract}
Chemical entropy generation and magnetohydrodynamic effects on the unsteady heat and fluid flow through a porous medium have been numerically investigated. The entropy generation due to the use of a magnetic field and porous medium effects on heat transfer, fluid friction, and mass transfer have been analyzed numerically. Using a similarity transformation, the governing equations of continuity, momentum, and energy and concentration equations, of nonlinear system, were reduced to a set of ordinary differential equations and solved numerically. The effects of unsteadiness parameter, magnetic field parameter, porosity parameter, heat generation/absorption parameter, Lewis number, chemical reaction parameter, and Brinkman number parameter on the velocity, the temperature, the concentration, and the entropy generation rates profiles were investigated and the results were presented graphically.
\end{abstract}

\section{Introduction}

Industries developing technology related to heat transfer are more concerned with the design of new thermal systems; thus research is in progress to investigate the hydrodynamic and heat transfer behavior of new forms of heat transfer fluid. Recently, entropy generation (or production) has been used to gauge the significance of irreversibility related to heat transfer, friction, and other nonideal processes within thermal system by Bejan [1]. Entropy generation and its minimization have been considered as an effective tool to improve the performance of any heat transfer process. Entropy generation minimization of diabetic distillation column with trays has been investigated using a new approach by Spasojević et al. [2] in which the exchanged heat has been considered as a control variable instead of temperature.

Andersson et al. [3] analyzed the momentum and heat transfer in a laminar liquid film on a horizontal stretching sheet governed by time-dependent boundary layer equations. Tsai et al. [4] studied the nonuniform heat source/sink effect on the flow and heat transfer from an unsteady stretching sheet through a quiescent fluid medium extending to infinity. Elbashbeshy and Bazid [5] presented similarity solutions of the boundary layer equations, which describe the unsteady flow and heat transfer over an unsteady stretching sheet. Shateyi and Motsa [6] investigated thermal radiation effects on heat and mass transfer over an unsteady stretching surface. Bouabid et al. [7] studied analysis of the magnetic field effect on entropy generation at thermosolutal convection in a square cavity. Oliveski et al. [8] proposed an entropy generation and natural convection in rectangular cavities. Achintya [9] Analyzed the entropy generation due to natural convection in square enclosures with multiple discrete heat sources. Magherbi et al. [10] investigated second law analysis in convective heat and mass transfer. El Jery et al. [11] studied effect of external oriented magnetic field on entropy generation in natural convection and Abd El-Aziz [12] studied radiation effect on the flow and heat transfer over an unsteady stretching sheet.

This study is a complementary study to the work of [6] to include the thermal radiation effects on heat and mass transfer over an unsteady stretching surface. This also 
presents chemical entropy generation and magnetohydrodynamic effects on the unsteady heat and fluid flow through a porous medium which are numerically investigated. The entropy generation due to the use of a magnetic field and porous medium effects on heat transfer, fluid friction, and mass transfer have been analyzed numerically. The effects of unsteadiness parameter, magnetic field parameter, porosity parameter, heat generation/absorption parameter, Lewis number, chemical reaction parameter, and Brinkman number parameter on the velocity, the temperature, the concentration, and the entropy generation rates profiles were investigated and the results were presented graphically.

\section{Problems Development}

The flow, assumed to be unsteady, laminar, and incompressible fluid on a horizontal sheet with chemical entropy generation and magnetohydrodynamic effects through a porous medium, has been considered. The viscous dissipation effect is also taken into consideration. The radiative heat flux in the $x$-direction is negligible in comparison with that in the $y$-direction. The fluid motion arises due to the stretching of the elastic sheet. The continuous sheet aligned with the $x$ axis at $y=0$ moves in its own plane with a surface velocity $U_{w}(x, t)$, the surface temperature $T_{w}(x, t)$, and the surface concentration $C_{w}(x, t)$ varying both along the sheet and with time. The magnetic Reynolds number of the flow is taken to be small enough that the induced magnetic field is assumed to be negligible in comparison with the applied magnetic field, that is, $B=\left(0, B_{0}, 0\right)$, where $B_{0}$ is the uniform magnetic field acting normal to the plate. $u$ and $v$ are the velocity of $x$ and $y$ component, and $T$ and $C$ are the temperature and concentration, respectively. The governing boundary layer equations of continuity, momentum, energy, and concentration equations under Boussinesq approximations could be written as follows.

The continuity equation:

$$
\frac{\partial u}{\partial x}+\frac{\partial v}{\partial y}=0
$$

The momentum equations:

$$
\frac{\partial u}{\partial t}+u \frac{\partial u}{\partial x}+v \frac{\partial u}{\partial y}=\nu \frac{\partial^{2} u}{\partial y^{2}}-\frac{\sigma B^{2}(x, t)}{\rho} u-S_{1}(x, t) u .
$$

The energy equation:

$$
\begin{aligned}
\frac{\partial T}{\partial t}+u \frac{\partial T}{\partial x}+v \frac{\partial T}{\partial y}= & \alpha \frac{\partial^{2} T}{\partial y^{2}}-\frac{1}{\rho C_{p}} \frac{\partial q_{r}}{\partial y} \\
& +\frac{Q_{1}(x, t)}{\rho C_{p}}\left(T-T_{\infty}\right)
\end{aligned}
$$

The concentration equations:

$$
\frac{\partial C}{\partial t}+u \frac{\partial C}{\partial x}+v \frac{\partial C}{\partial y}=D \frac{\partial^{2} C}{\partial y^{2}}-k_{1}(x, t)\left(C-C_{\infty}\right) .
$$

The boundary conditions are

$$
\begin{gathered}
u(x, 0)=U_{w}(x, t), \\
v(x, 0)=0, \\
T(x, 0)=T_{\omega}(x, t), \\
C(x, 0)=C_{w}(x, t), \\
u(x, \infty) \longrightarrow 0, \\
T(x, \infty) \longrightarrow T_{\infty}, \\
C(x, \infty) \longrightarrow C_{\infty},
\end{gathered}
$$

where $\sigma$ is the electrical conductivity, $\rho$ is the density of the fluid, $\mu$ is the viscosity of the fluid, $\alpha=k / \rho C_{p}$ is the thermal diffusivity of the fluid, $C_{p}$ is the heat capacity at constant pressure, $k_{1}$ is the thermal conductivity, $Q_{1}$ is heat generation/absorption rate (is positive in the case of the sheet's generation of heat and is negative in the case of the sheet's absorption of heat from the fluid flow), $D$ is the mass diffusivity, $T_{\infty}$ is temperature of the fluid at infinity, and $q_{r}$ is the radiative heat flux in the $y$-direction. Using the Rosseland approximation (Sparrow and Cess [13] and Moradi et al. [14]), the radiative heat flux $q_{r}$ is given by

$$
q_{r}=-\frac{4 \sigma^{*}}{3 k^{*}} \frac{\partial T^{4}}{\partial y}
$$

where $\sigma^{*}$ is the Stefan-Boltzmann constant and $k^{*}$ is the mean absorption coefficient. Assuming that the temperature difference within the flow is sufficiently small such that $T^{4}$ could be approached as the linear function of temperature:

$$
T^{4} \cong 4 T_{\infty}^{3} T-3 T_{\infty}^{4} .
$$

Following Andersson et al. [15], the surface velocity $U_{w}(x, t)$ is assumed to be $U_{w}(x, t)=b x /(1-a t)$, where both $a$ and $b$ are positive constants with dimension reciprocal time. We have $b$ as the initial stretching rate and $b /(1-a t)$ is increasing with time. In the context of polymer extrusion, the material properties particularly the elasticity of the extruded sheet may vary with time even though the sheet is being pulled by a constant force. With unsteady stretching, however, $a^{-1}$ becomes the representative time scale of the resulting unsteady boundary layer problem. We assume that all of the surface temperature $T_{w}(x, t)$, the surface concentration $C_{w}(x, t)$, the applied transverse magnetic field $B(x, t)$, the volumetric heat generation/absorption rate $Q_{1}(x, t)$, the thermal conductivity $k_{1}(x, t)$, and the porous medium $S_{1}(x, t)$ are on 
a stretching sheet to vary with the distance $x$ along the sheet and time in the following forms:

$$
\begin{aligned}
B(x, t) & =B_{0}(1-a t)^{-1 / 2}, \\
Q_{1}(x, t) & =Q_{0}(1-a t)^{-1}, \\
T_{w}(x, t) & =T_{\infty}+T_{0}\left(\frac{b x^{2}}{2 \nu}\right)(1-a t)^{-3 / 2}, \\
C_{w}(x, t) & =C_{\infty}+C_{0}\left(\frac{b x^{2}}{2 \nu}\right)(1-a t)^{-3 / 2}, \\
S_{1}(x, t) & =\frac{\nu}{\kappa}=S_{0}(1-a t)^{-1}, \\
k_{1}(x, t) & =k_{0}(1-a t)^{-1} .
\end{aligned}
$$

We introduce the similarity transformations

$$
\begin{aligned}
& \psi=\sqrt{v b}(1-a t)^{-1 / 2} x f(\eta), \\
& T=T_{\infty}+T_{0}\left(\frac{b x^{2}}{2 v}\right)(1-a t)^{-3 / 2} \theta(\eta), \\
& C=C_{\infty}+C_{0}\left(\frac{b x^{2}}{2 \nu}\right)(1-a t)^{-3 / 2} \varphi(\eta), \\
& \eta=\sqrt{\frac{b}{v}}(1-a t)^{-1 / 2} y .
\end{aligned}
$$

Equation (1) is satisfied automatically and so are governing equations (2)-(5); we have

$$
\begin{aligned}
& f^{\prime \prime \prime}-A\left(\frac{\eta f^{\prime \prime}}{2}+f^{\prime}\right)+f f^{\prime \prime}-f^{\prime 2}-(M+S) f^{\prime}=0 \\
& (3 R+4) \theta^{\prime \prime} \\
& \quad+3 R P_{r}\left(f \theta^{\prime}-2 f^{\prime} \theta-\left(\frac{A}{2}\right)\left(3 \theta+\eta \theta^{\prime}\right)+H \theta\right) \\
& \quad=0 \\
& \varphi^{\prime \prime}+P_{r} L_{e}\left(f \varphi^{\prime}-2 f^{\prime} \varphi-\left(\frac{A}{2}\right)\left(3 \varphi+\eta \varphi^{\prime}\right)-\gamma \varphi\right) \\
& \quad=0
\end{aligned}
$$

with the boundary conditions

$$
\begin{gathered}
f(0)=0, \\
f^{\prime}(0)=1, \\
\theta(0)=1, \\
\varphi(0)=1, \\
f^{\prime}(\infty)=0, \\
\theta(\infty)=0, \\
\varphi(\infty)=0 .
\end{gathered}
$$

Here the prime denotes a partial differentiation with respect to $\eta, \kappa$ is the permeability of the porous medium, $A=a / b$ is the unsteadiness parameter, $M=\sigma B_{0}^{2} / b \rho$ is the magnetic field parameter, $S=S_{0} / b$ is the porosity parameter, $P_{r}=\mu C_{p} \nu / k$ is the Prandtl number, $R=k^{*} k / 4 \sigma^{*} T_{\infty}^{3}$ is the thermal radiation parameter, $H=Q_{0} / b \rho C_{p}$ is the heat generation/absorption parameter, $L_{e}=k / D \rho C_{p}$ is the Lewis number, and $\gamma=k_{0} / b$ is the chemical reaction parameter.

\section{Entropy Generation}

In the present problem, the volumetric entropy generation is therefore the sum of irreversibilities due to heat transfer, fluid friction, mass transfer by pure concentration gradients, and mass transfer by mixed product of concentration and thermal gradients with magnetic field and porous medium effects which is given by

$$
\begin{aligned}
S_{G}= & \frac{k}{T_{\infty}^{2}}\left(\frac{\partial T}{\partial y}\right)^{2}+\frac{\mu}{T_{\infty}}\left(\frac{\partial u}{\partial y}\right)^{2}+\frac{\mu}{C_{\infty}}\left(\frac{\partial C}{\partial y}\right)^{2} \\
& +\frac{\mu}{C_{\infty}}\left(\frac{\partial T}{\partial y}\right)\left(\frac{\partial C}{\partial y}\right)+\frac{\sigma B_{0}^{2}}{T_{\infty}} u^{2}+\frac{\mu}{\kappa T_{\infty}} u^{2} .
\end{aligned}
$$

Using the nondimensional quantities, we obtain the local entropy generation rates in nondimensional form:

$$
\begin{aligned}
N_{s}= & \lambda_{1} \frac{k}{T_{\infty}^{2}}\left(\frac{\partial T}{\partial y}\right)^{2}+\lambda_{2} \frac{\mu}{T_{\infty}}\left(\frac{\partial u}{\partial y}\right)^{2} \\
& +\lambda_{3} \frac{\mu}{C_{\infty}}\left(\frac{\partial C}{\partial y}\right)^{2}+\lambda_{4} \frac{\mu}{C_{\infty}}\left(\frac{\partial T}{\partial y}\right)\left(\frac{\partial C}{\partial y}\right) \\
& +\lambda_{5} \frac{\sigma B_{0}^{2}}{T_{\infty}} u^{2}+\lambda_{6} \frac{\mu}{\kappa T_{\infty}} u^{2},
\end{aligned}
$$

where $\lambda_{1}=x^{2} T_{\infty}^{2}(1-a t)^{4} / k(\Delta T)^{2}, \lambda_{2}=x^{2} T_{\infty}^{2}(1-a t)^{3} /$ $k(\Delta T)^{2}, \lambda_{3}=T_{\infty}(\Delta C / \Delta T)^{2}(1-a t)^{4} / k, \lambda_{4}=T_{\infty}(\Delta C / \Delta T)(1-$ at $)^{4} / k, \lambda_{5}=x^{2} T_{\infty}(1-a t)^{2} / k(\Delta T)^{2}$, and $\lambda_{6}=x^{2} T_{\infty}(1-$ at $)^{3} / k(\Delta T)^{2}$ are the characteristic entropy generation rate.

The total dimensionless entropy generation is obtained by

$$
\begin{aligned}
N_{s} & =B_{r} R_{e}\left[\theta^{\prime 2}+f^{/ / 2}+\frac{\Omega_{1}}{\Omega} \varphi^{\prime 2}+\Omega_{1} \theta^{\prime} \varphi^{\prime}\right. \\
& \left.+\frac{1}{\Omega}(M+S) f^{/ 2}\right],
\end{aligned}
$$

where $B_{r}=\mu T_{\infty} b^{2} x^{2} / k(\Delta T)$ is the Brinkman number, $R_{e}=b x^{2} / \nu$ is local Reynolds number, $\Omega=\Delta T / T_{\infty}$ is the dimensionless temperature ratio, and $\Omega_{1}=\Delta C / C_{\infty}$ is the dimensionless concentration ratio.

\section{Result and Discussion}

The system of nonlinear ordinary differential equations (10) together with the boundary conditions (11) is locally similar and solved numerically by using the Control Volume FiniteElement Method. Numerical values of the velocity, the temperature, and the concentration profiles have been used to 


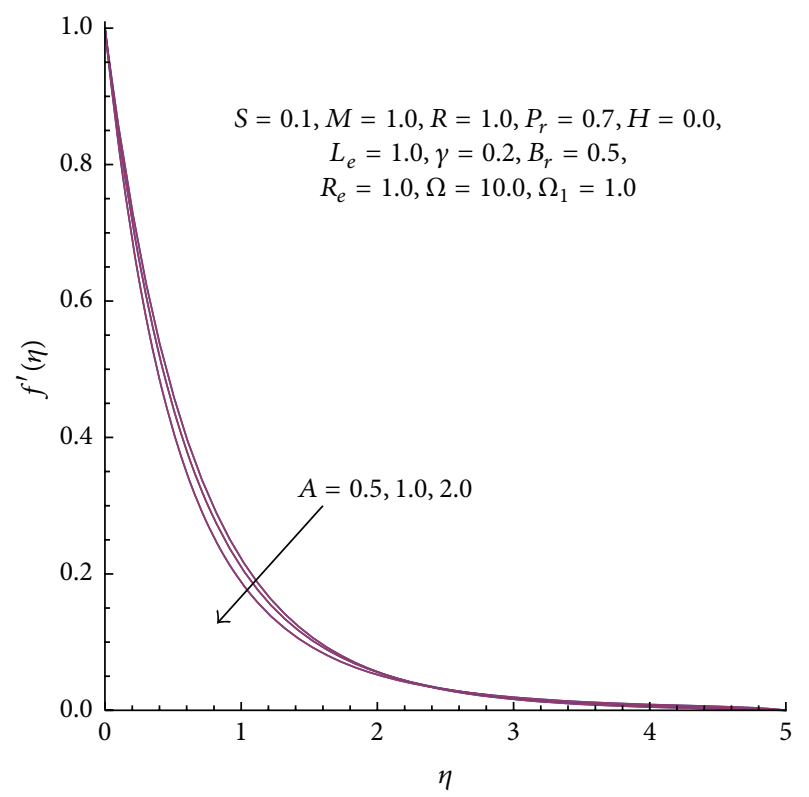

(a)

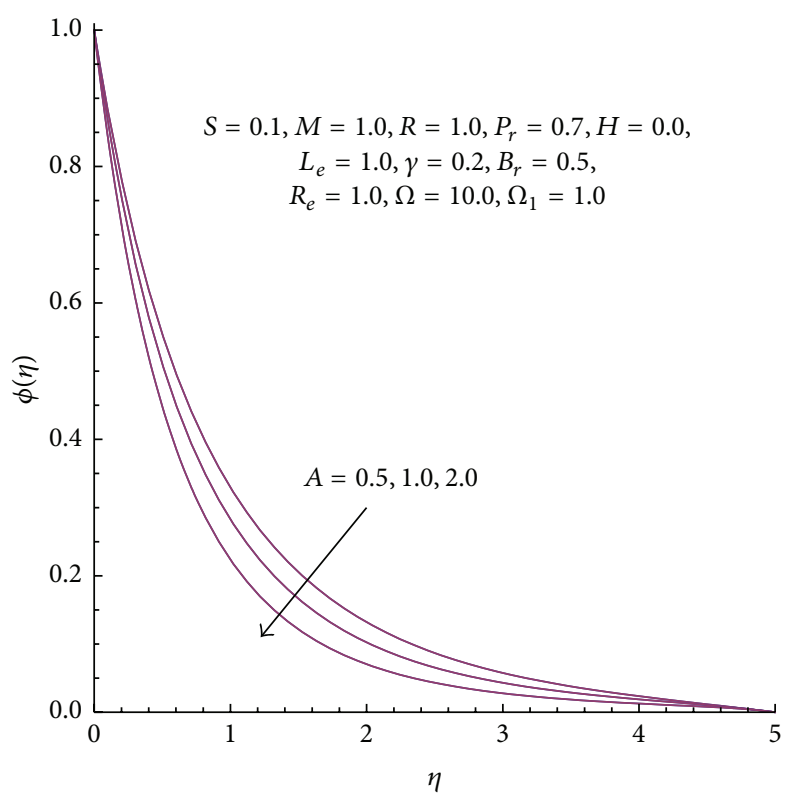

(c)

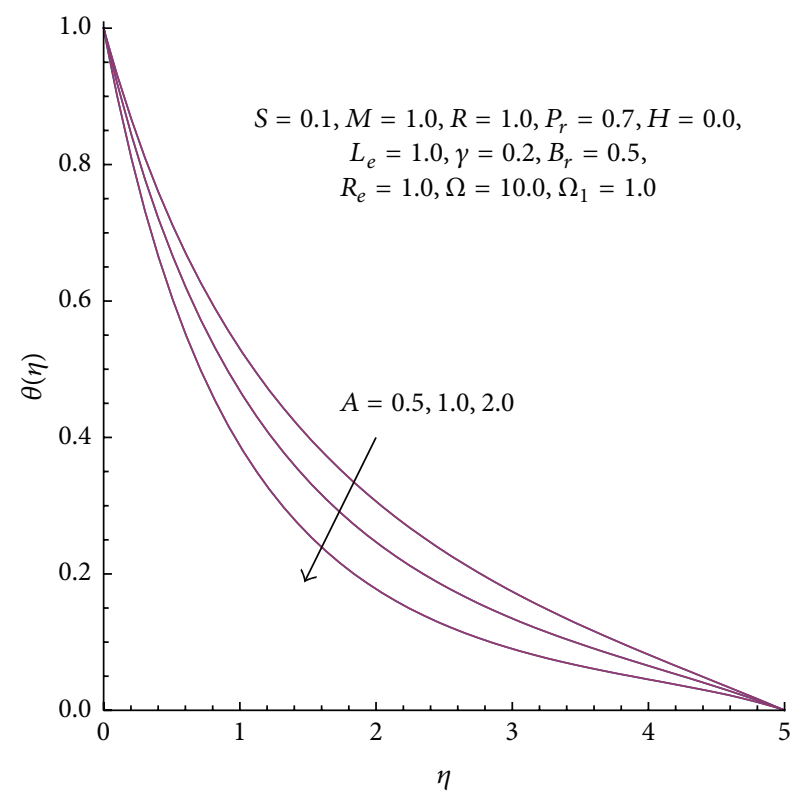

(b)

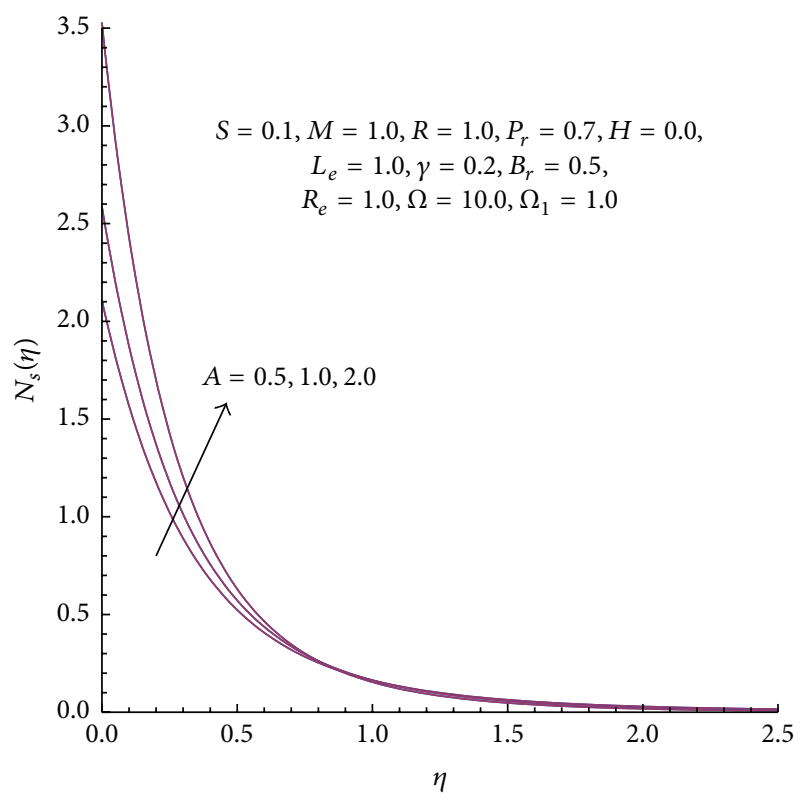

(d)

FIGURE 1: Influence of unsteadiness parameter on (a) the velocity profile, (b) the temperature profile, (c) the concentration profile, and (d) the entropy generation rates profile.

compute the entropy generation rates, found for different values of the various parameters occurring in the problem with unsteadiness parameter, magnetic field parameter, porosity parameter, heat generation/absorption parameter, Lewis number, chemical reaction parameter, and Brinkman number parameter; the results are displayed in Figures 17, for the velocity, the temperature, the concentration, and the entropy generation rates profiles. In order to verify the accuracy of our present method, we have compared our results with those of Shateyi and Motsa [6] and Abd El-Aziz [12]. Table 1 shows the values of $-\theta^{\prime}(0)$ for several of $A$ and $P_{r}$.
The comparisons in all the above cases are found to agree with each other excellently. Also the results are found to be similar to those by Shateyi and Motsa [6] and Abd El-Aziz [12]. So it is good.

In Figures 1(a), 1(b), 1(c), and 1(d), respectively, the influence of the unsteadiness parameter on the velocity profile, the temperature profile, the concentration profile, and the entropy generation rates profile is shown. It is observed that the velocity profile, the temperature profile, and the concentration profile decrease while the entropy generation rates profile increases with the increase of the unsteadiness 


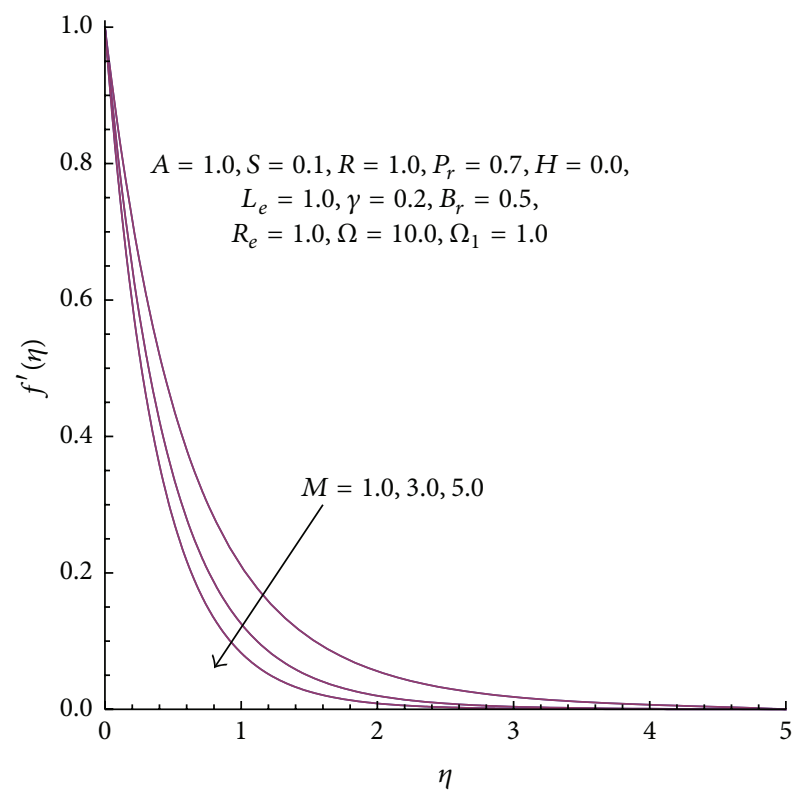

(a)

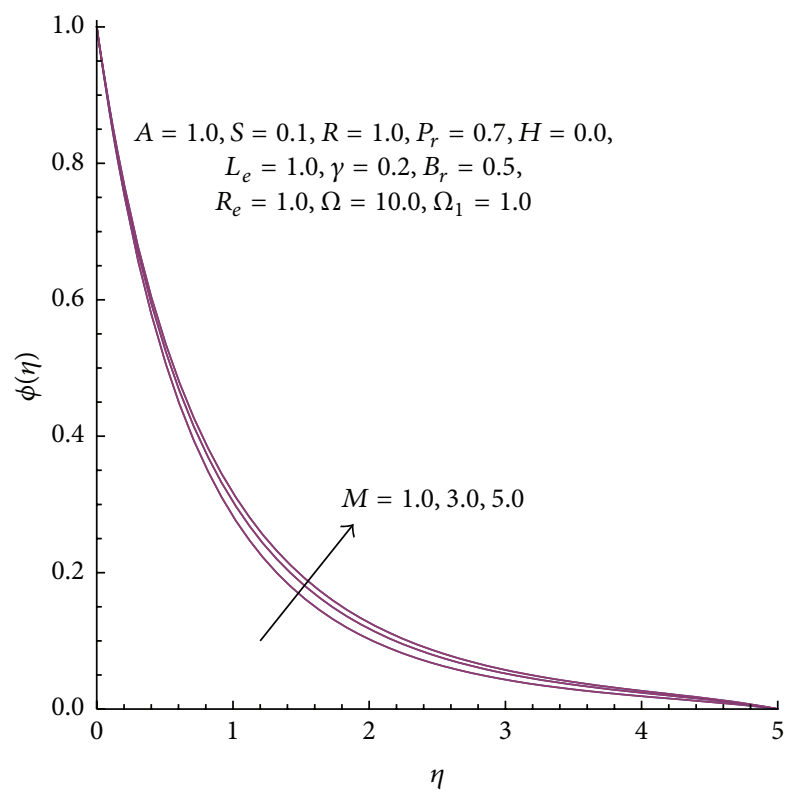

(c)

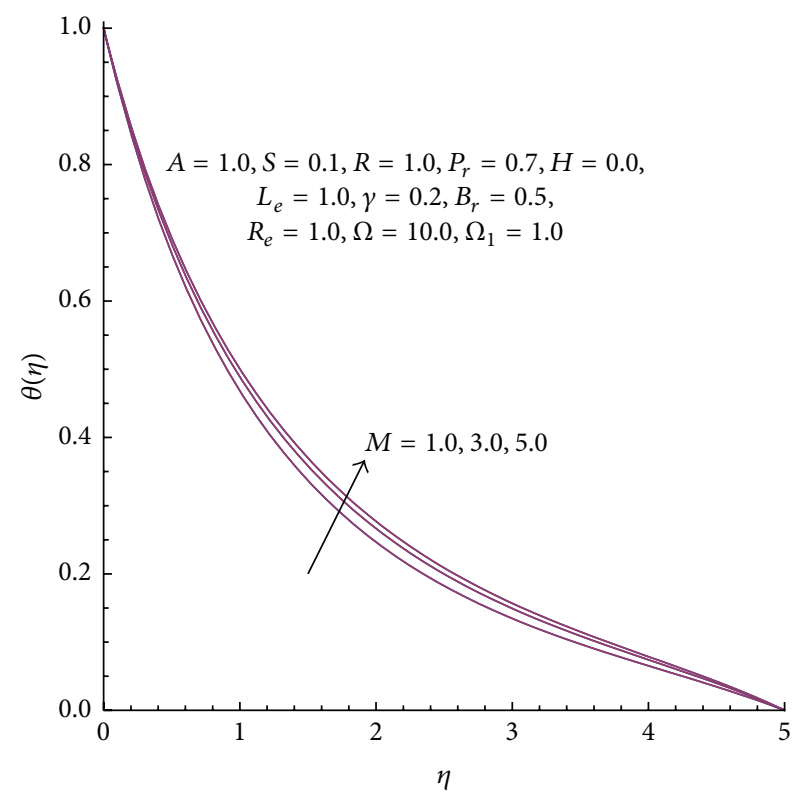

(b)

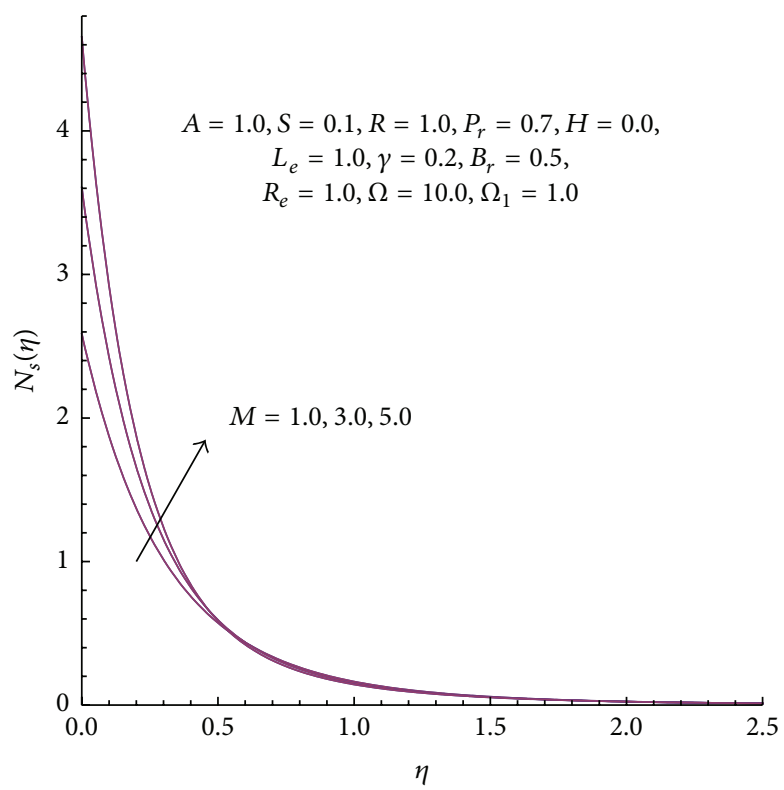

(d)

FIGURE 2: Influence of the magnetic field parameter on (a) the velocity profile, (b) the temperature profile, (c) the concentration profile, and (d) the entropy generation rates profile.

parameter. When $A=0$, we have a steady state flow and for $A>0$, we have an unsteady flow.

In absence of both magnetic field parameter and porosity parameter effects on the velocity profile, the temperature profile, the concentration profile, and the entropy generation rates profile are illustrated in Figures 2(a), 2(b), 2(c), 2(d), 3(a), 3(b), 3(c), and 3(d), respectively; also, we have found that the velocity profile decreases while the temperature profile, the concentration profile, and the entropy generation rates profile increase with the increase of each of the magnetic field parameter and porosity parameter, and this is due to the fact that the thermal boundary layer increases with magnetic field parameter and porosity parameter. The presence of each of the magnetic field and porosity creates additional entropy.

The effects of heat generation/absorption parameter on the temperature and the entropy generation rates profiles are presented in Figures 4(a) and 4(b), respectively. It is observed that the temperature profile increases while the entropy generation rates profile decreases with increase of heat generation/absorption parameter. This is due to the fact 


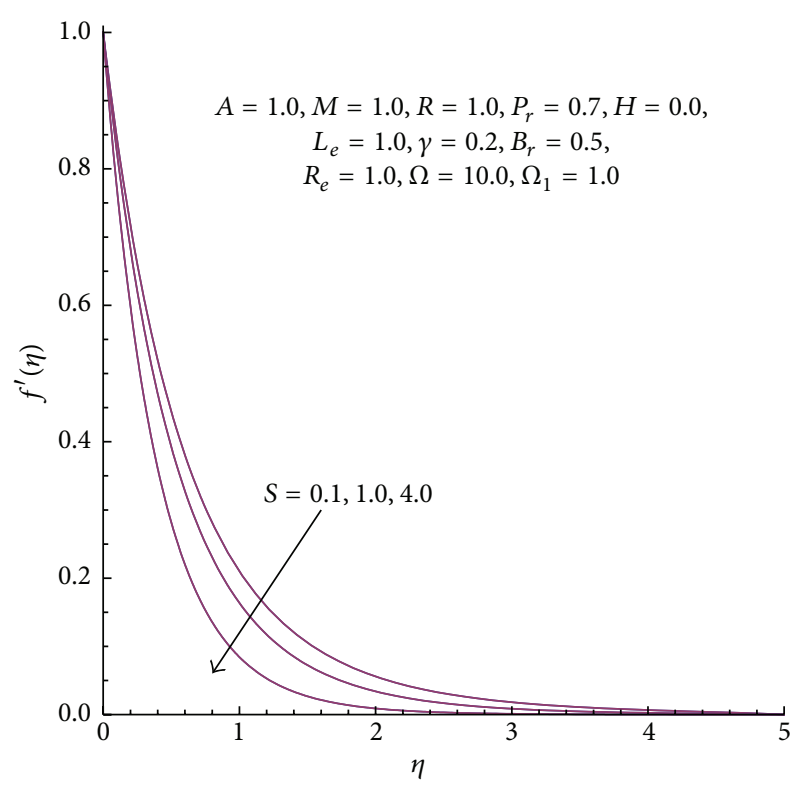

(a)

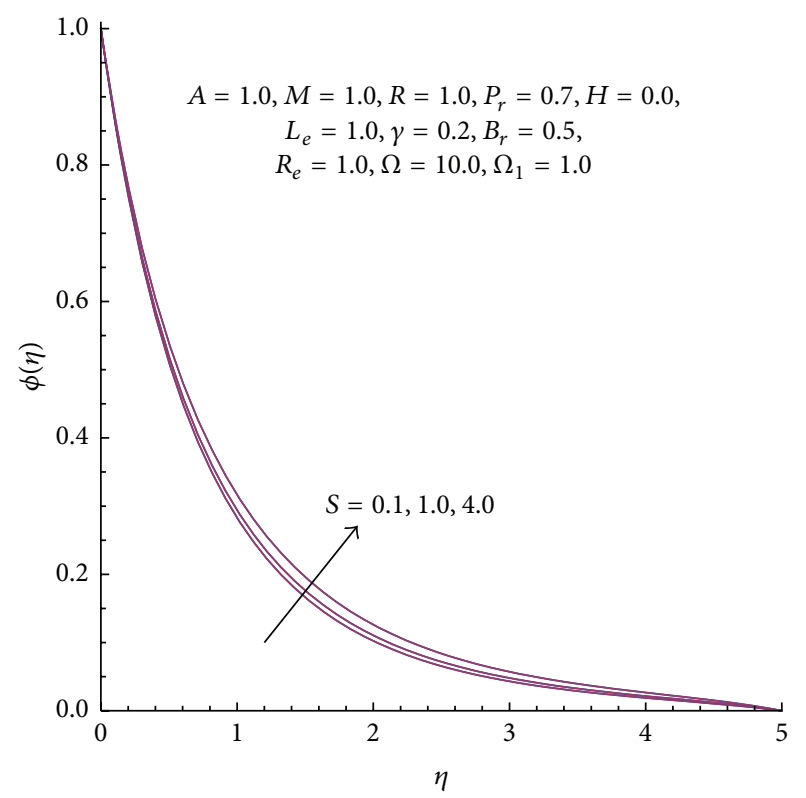

(c)

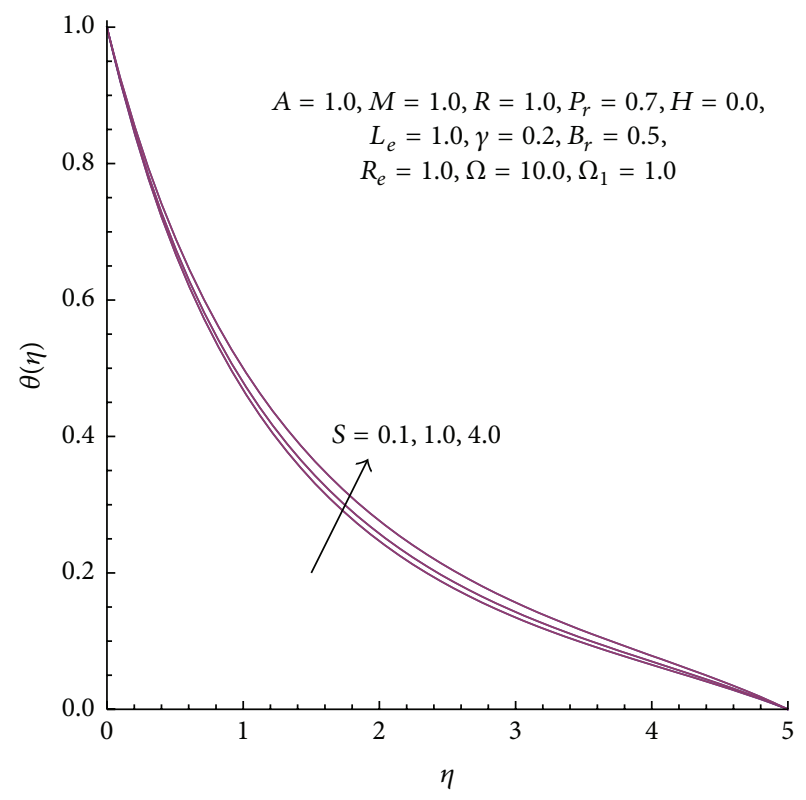

(b)

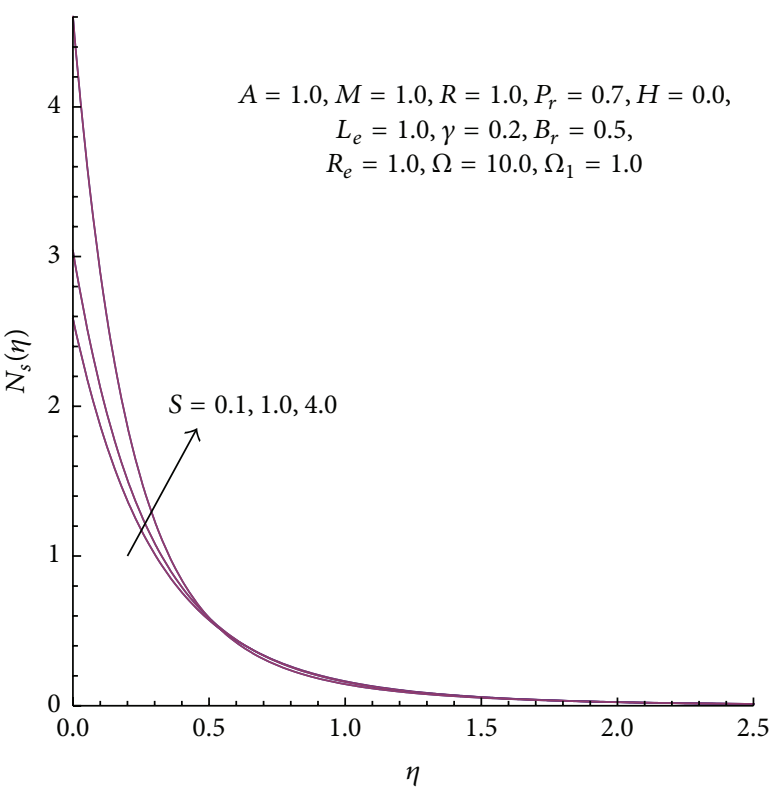

(d)

FIGURE 3: Influence of the porosity parameter on (a) the velocity profile, (b) the temperature profile, (c) the concentration profile, and (d) the entropy generation rates profile.

that the increase of the heat source/sink parameter means an increase of the heat generated inside the boundary layer leading to higher temperature profile.

The effect of the Lewis number parameter on the concentration and the entropy generation rates profiles is shown on Figures 5(a) and 5(b), respectively, and we have found that the concentration profile decreases while entropy generation rates profile increases with increase of the values of the Lewis number parameter.

The concentration profile for different values of the chemical reaction parameter is plotted in Figure 6; we observe that the concentration profile decreases with the increase of the chemical reaction parameter. The influence of the Brinkman number parameter on the entropy generation rates profile is shown in Figure 7; we have found that the entropy generation rates profile increases with the increase of the Brinkman number parameter.

\section{Conclusions}

The entropy generation due to the use of a magnetic field and porous medium effects on heat transfer, fluid friction, 


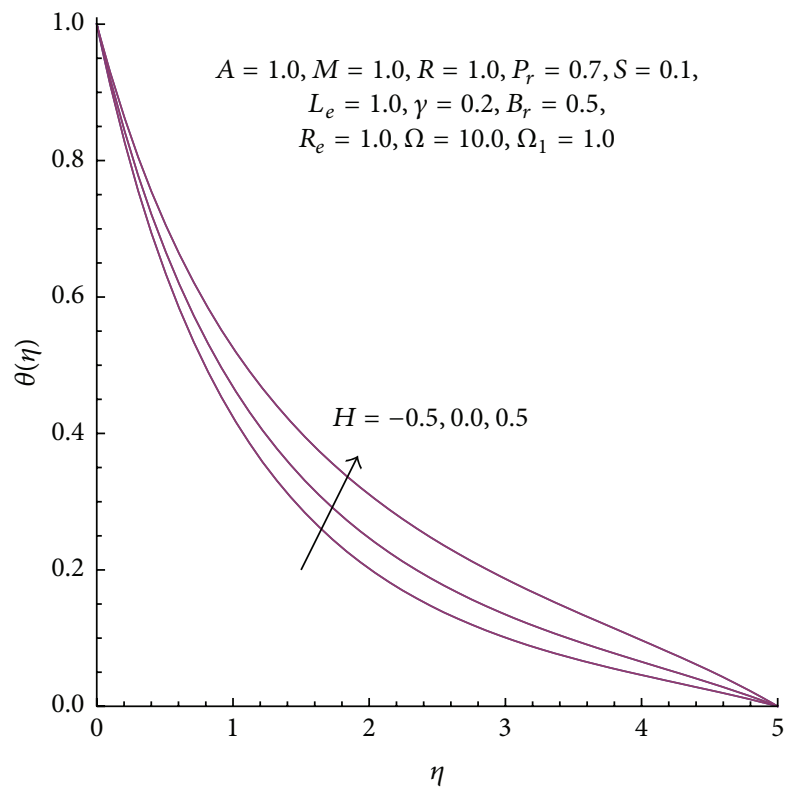

(a)

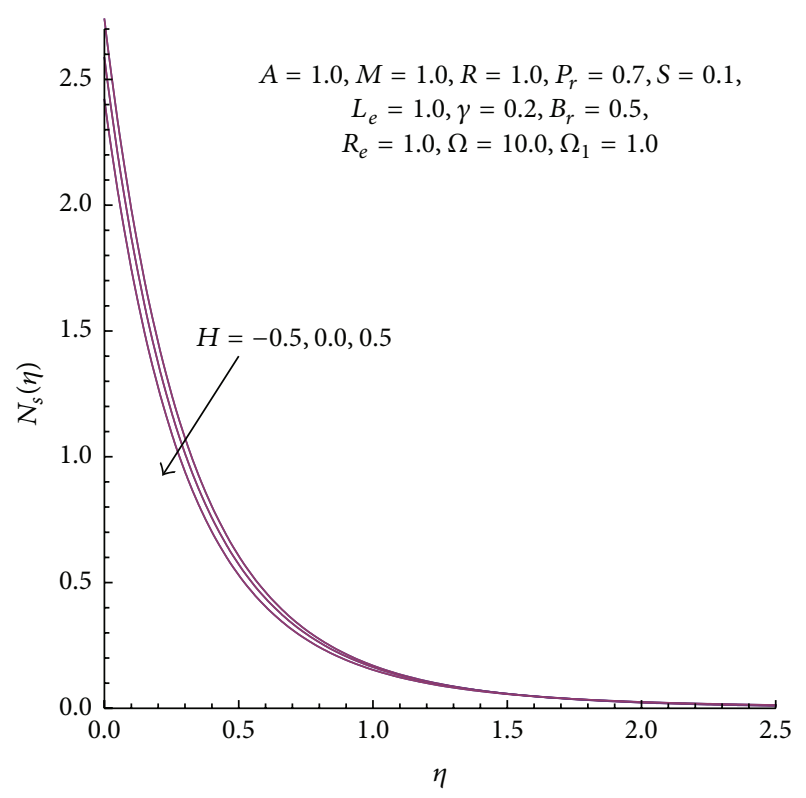

(b)

FIGURE 4: Influence of the heat generation/absorption parameter on (a) the temperature profile and (b) the entropy generation rates profile.

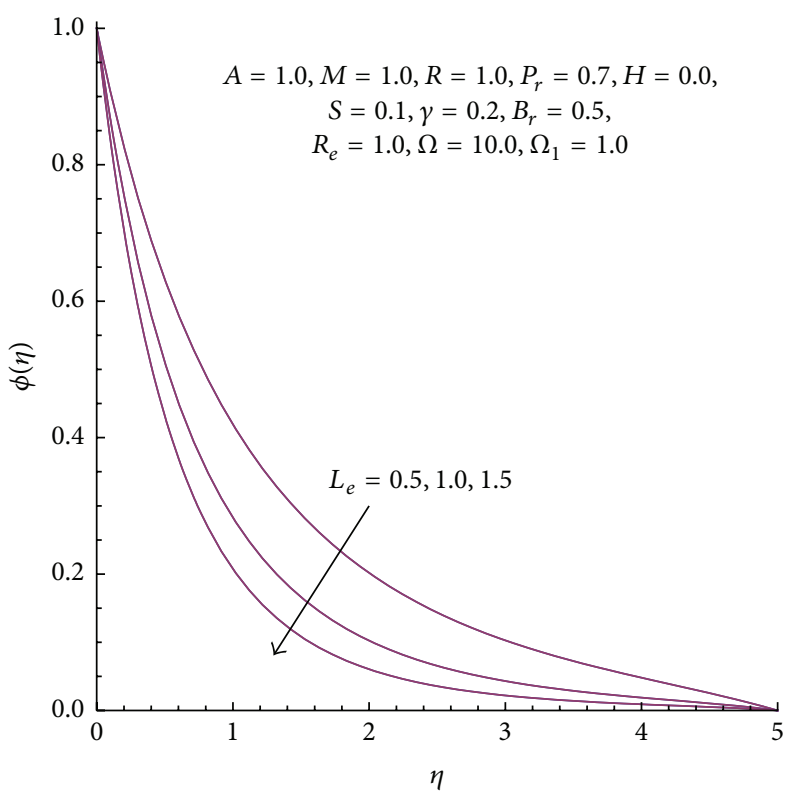

(a)

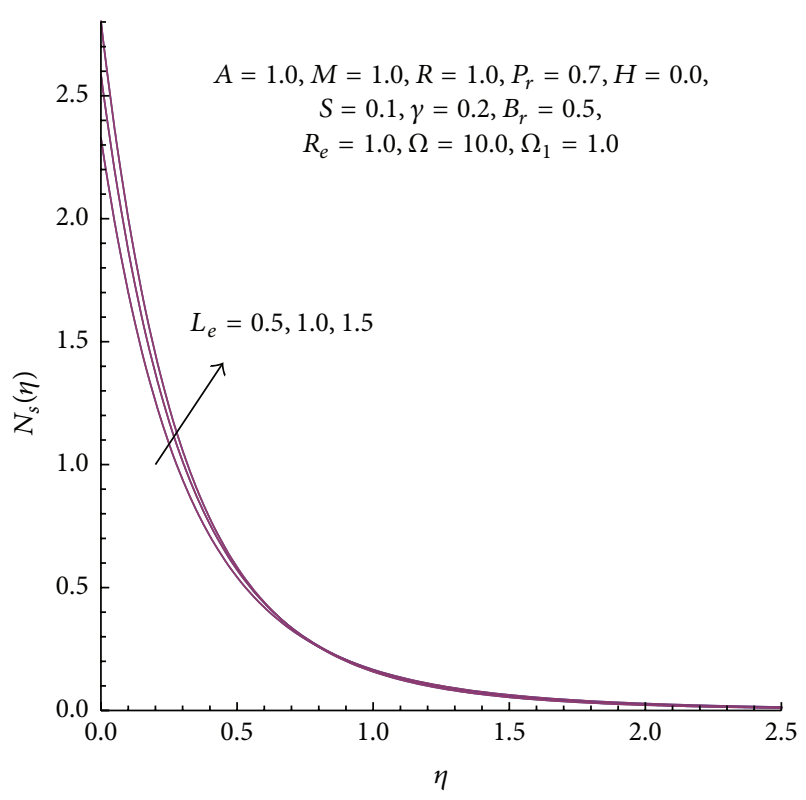

(b)

FIGURE 5: Influence of the Lewis number parameter on (a) the concentration profile and (b) the entropy generation rates profile.

and mass transfer have been analyzed numerically. Numerical values of the velocity, the temperature, and the concentration profiles have been used to compute the entropy generation rates, found for different values of the various parameters occurring in the problem. The effects of unsteadiness parameter, magnetic field parameter, porosity parameter, heat generation/absorption parameter, Lewis number, chemical reaction parameter, and Brinkman number parameter on the velocity, the temperature, the concentration, and the entropy generation rates profiles are discussed graphically. The main conclusions derived from this study are given below:

(1) The increasing values of porosity parameter and magnetic field parameter lead to increase in the entropy generation rates profile, and we also observed that the temperature profile and the concentration profile increase, while the velocity profile decreases. 


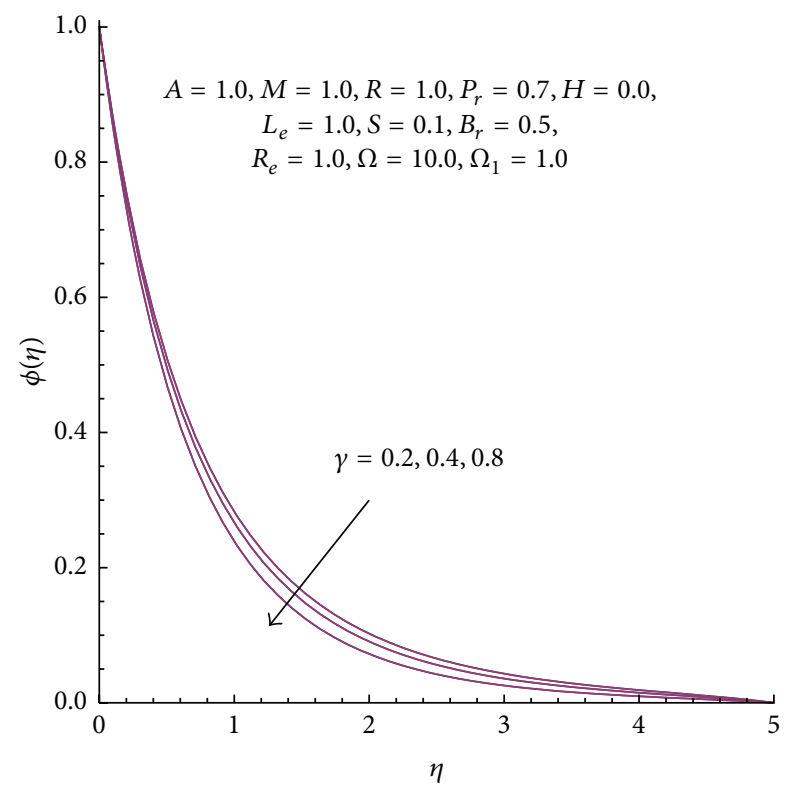

FIGURE 6: Influence of the chemical reaction parameter on the concentration profile.

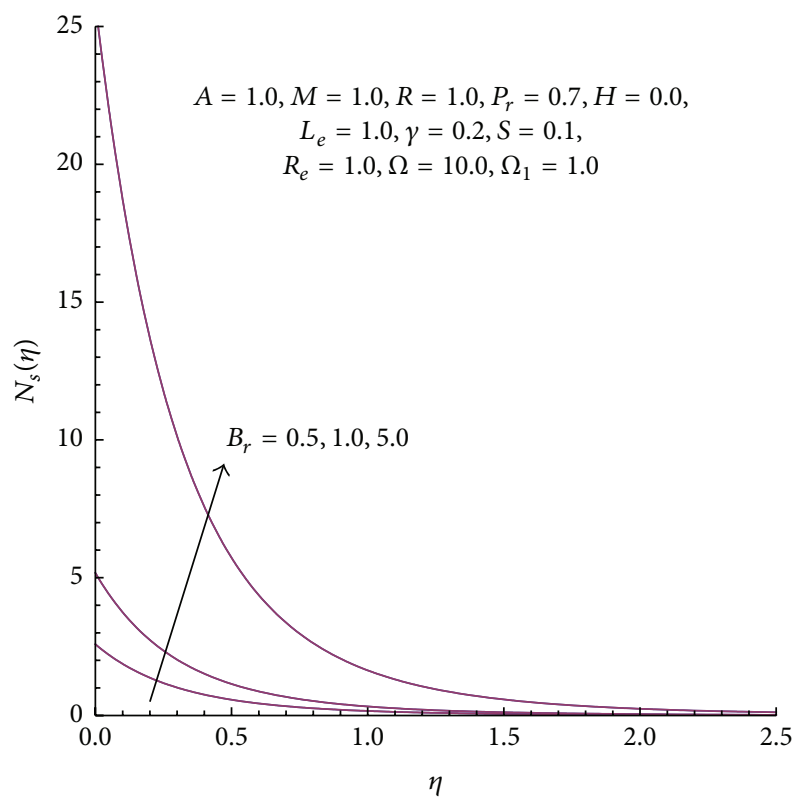

FIGURE 7: Influence of the Brinkman number parameter on the entropy generation rates profile.

(2) The entropy generation rates profile decreases with the increase of the heat generation/absorption parameter. And also, the temperature profile increases.

(3) As the Lewis number parameter increases, we found that the entropy generation rates profile increases, while the concentration profile decreases.
TABLE 1: Comparison of the value $-\theta^{\prime}(0)$ for several of $A$ and $P_{r}$ with $M=0.0, S=0.0, H=0.0, L_{e}=0.0, \gamma=0.0$, and $B_{r}=0.0$.

\begin{tabular}{ccccc}
\hline$A$ & $P_{r}$ & Abd El-Aziz [12] & Shateyi and Motsa [6] & Present study \\
\hline & 0.1 & 0.4517 & 0.45149 & 0.4518 \\
0.8 & 1.0 & 1.6728 & 1.67285 & 1.67275 \\
& 10.0 & 5.70503 & 5.70598 & 5.70573 \\
\hline & 0.1 & 0.5087 & 0.50850 & 0.50841 \\
1.2 & 1.0 & 1.818 & 1.81801 & 1.81793 \\
& 10.0 & 6.12067 & 6.12102 & 6.12012 \\
\hline & 0.1 & 0.606013 & 0.60352 & 0.60341 \\
2.0 & 1.0 & 2.07841 & 2.07841 & 2.07830 \\
& 10.0 & 6.88506 & 6.88615 & 6.88586 \\
\hline
\end{tabular}

(4) The entropy generation rates profile decreases with the increase of the Brinkman number parameter.

(5) The unsteadiness parameter increases with the increase of the entropy generation rates profile, and also, the velocity profile, the temperature profile, and the concentration profile decrease.

\section{Nomenclature}

A: Unsteadiness parameter $(=a / b)$

a: Positive constant

$B$ : Magnetic field

$B_{0}$ : Uniform transverse magnetic field

$B_{r}:$ Brinkman number $\left(=\mu T_{\infty} b^{2} x^{2} / k(\Delta T)\right)$

$b$ : Positive constant

$C:$ Concentration profile

$C_{p}$ : Heat capacity at constant pressure

$C_{w}$ : Surface concentration

$D$ : Mass diffusivity

$H$ : Heat generation/absorption parameter $\left(=Q_{0} / b \rho C_{p}\right)$

$k$ : Thermal conductivity

$k^{*}$ : Mean absorption coefficient

$L_{e}:$ Lewis number $\left(=k / D \rho C_{p}\right)$

$M:$ Magnetic field parameter $\left(=\sigma B_{0}^{2} / b \rho\right)$

$N_{s}$ : Entropy generation rates

$Q_{1}$ : Volumetric heat generation/absorption rate

$Q_{0}$ : Heating source

$q_{r}$ : Radiative heat flux

$T: \quad$ Temperature profile

$T_{w}$ : Surface temperature

$T_{\infty}$ : Temperature of the fluid at infinity

$S: \quad$ Porosity parameter $\left(=S_{0} / b\right)$

$S_{G}$ : Volumetric entropy generation

$P_{r}:$ Prandtl number $\left(=\mu C_{p} \nu / k\right)$

$R: \quad$ Thermal radiation parameter $\left(=k^{*} k / 4 \sigma^{*} T_{\infty}^{3}\right)$

$R_{e}:$ Local Reynolds number $\left(=b x^{2} / \nu\right)$

$u$ : Velocity in the $x$-direction

$U_{w}$ : Surface velocity

$v$ : Velocity in the $y$-direction

$x$ : Horizontal distance

$y$ : Vertical distance. 


$\begin{array}{ll}\text { Greek Symbols } \\ \lambda_{1}, \lambda_{2}, \lambda_{3}, \lambda_{4}, \lambda_{5}: \begin{array}{l}\text { Characteristic entropy generation rate } \\ \text { Thermal diffusivity of the fluid } \\ \left(=k / \rho C_{p}\right)\end{array} \\ \gamma: & \begin{array}{l}\text { Chemical reaction parameter }\left(=k_{0} / b\right) \\ \text { Dimensionless temperature ratio } \\ \left(=\Delta T / T_{\infty}\right)\end{array} \\ & \begin{array}{l}\text { Dimensionless concentration ratio } \\ \left(=\Delta C / C_{\infty}\right)\end{array} \\ \Omega_{1}: & \text { Similarity variable } \\ \eta: & \text { Dimensionless temperature distribution } \\ \theta: & \text { Stream function } \\ \psi: & \text { Viscosity of the fluid } \\ \mu: & \text { Density of the fluid } \\ \rho: & \text { Kinematic viscosity of the fluid } \\ \nu: & \text { Permeability of the porous medium } \\ \kappa: & \text { Electrical conductivity } \\ \sigma: & \text { Stephan-Boltzmann constant. }\end{array}$

\section{Subscripts}

$w, \infty$ : Conditions at the surface and in the free stream.

\section{Conflict of Interests}

The author declares that there is no conflict of interests regarding the publication of this paper.

\section{References}

[1] A. Bejan, Entropy Generation through Heat and Fluid Flow, John Wiley \& Sons, New York, NY, USA, 1982.

[2] M. D. Spasojević, M. R. Janković, and D. D. Djaković, "A new approach to entropy production minimization in diabatic distillation column with trays," Thermal Science, vol. 14, no. 2 , pp. 317-328, 2010.

[3] H. I. Andersson, J. B. Aarseth, and B. S. Dandapat, "Heat transfer in a liquid film on an unsteady stretching surface," International Journal of Heat and Mass Transfer, vol. 43, no. 1, pp. 69-74, 2000.

[4] R. Tsai, K. H. Huang, and J. S. Huang, "Flow and heat transfer over an unsteady stretching surface with non-uniform heat source," International Communications in Heat and Mass Transfer, vol. 35, no. 10, pp. 1340-1343, 2008.

[5] E. M. Elbashbeshy and M. A. Bazid, "Heat transfer over an unsteady stretching surface with internal heat generation," Applied Mathematics and Computation, vol. 138, no. 2-3, pp. 239-245, 2003.

[6] S. Shateyi and S. S. Motsa, "Thermal radiation effects on heat and mass transfer over an unsteady stretching surface," Mathematical Problems in Engineering, vol. 2009, Article ID 965603, 13 pages, 2009.

[7] M. Bouabid, N. Hidouri, M. Magherbi, and A. Ben Brahim, "Analysis of the magnetic field effect on entropy generation at thermosolutal convection in a square cavity," Entropy, vol. 13, no. 5, pp. 1034-1054, 2011.

[8] R. D. C. Oliveski, M. H. Macagnan, and J. B. Copetti, "Entropy generation and natural convection in rectangular cavities," Applied Thermal Engineering, vol. 29, no. 8-9, pp. 1417-1425, 2009.
[9] M. Achintya, "Analysis of entropy generation due to natural convection in square enclosures with multiple discrete heat sources," International Communications in Heat and Mass Transfer, vol. 37, no. 7, pp. 867-872, 2010.

[10] M. Magherbi, H. Abbassi, N. Hidouri, and A. Ben Brahim, "Second law analysis in convective heat and mass transfer," Entropy, vol. 8, no. 1, pp. 1-17, 2006.

[11] A. El Jery, N. Hidouri, M. Magherbi, and A. B. Ben Brahim, "Effect of external oriented magnetic field on entropy generation in natural convection," Entropy, vol. 12, no. 6, pp. 1391-1417, 2010.

[12] M. Abd El-Aziz, "Radiation effect on the flow and heat transfer over an unsteady stretching sheet," International Communications in Heat and Mass Transfer, vol. 36, no. 5, pp. 521-524, 2009.

[13] E. M. Sparrow and R. D. Cess, Radiation Heat Transfer, Brooks/Cole, Belmont, Calif, USA, 1970.

[14] A. Moradi, H. Ahmadikia, T. Hayat, and A. Alsaedi, "On mixed convection-radiation interaction about an inclined plate through a porous medium," International Journal of Thermal Sciences, vol. 64, pp. 129-136, 2013.

[15] H. I. Andersson, J. B. Aarseth, N. Braud, and B. S. Dandapat, "Flow of a power-law fluid film on an unsteady stretching surface," Journal of Non-Newtonian Fluid Mechanics, vol. 62, no. 1, pp. 1-8, 1996. 


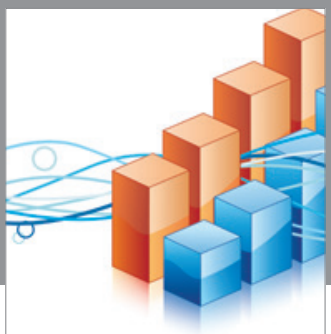

Advances in

Operations Research

vatem alat4

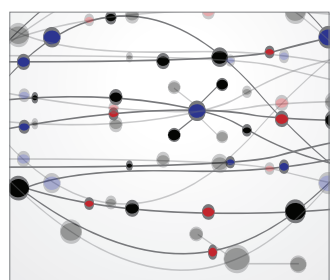

\section{The Scientific} World Journal
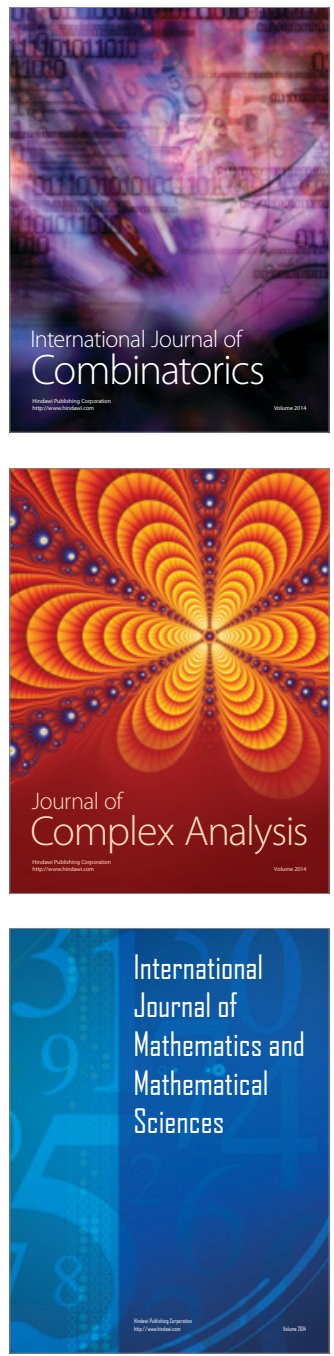
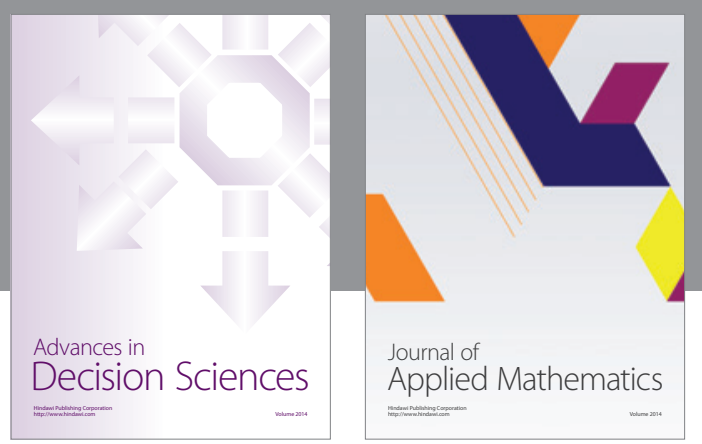

Algebra

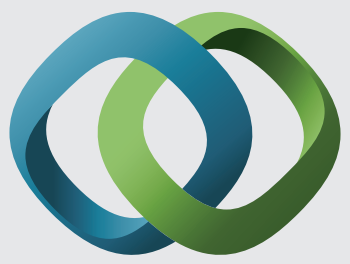

\section{Hindawi}

Submit your manuscripts at

http://www.hindawi.com
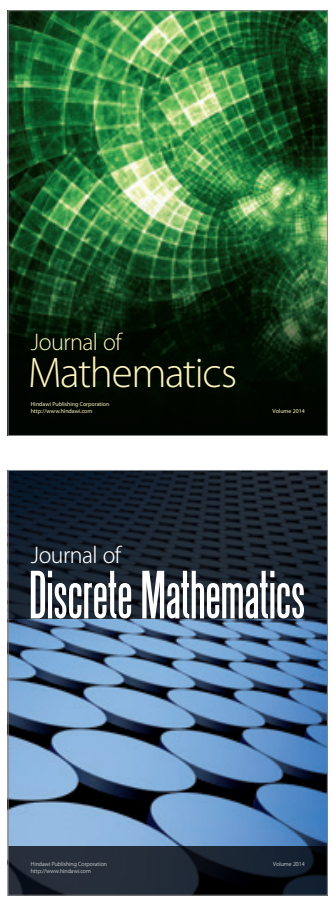

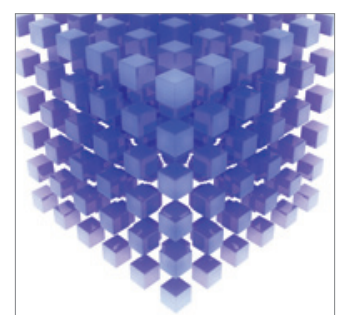

Mathematical Problems in Engineering
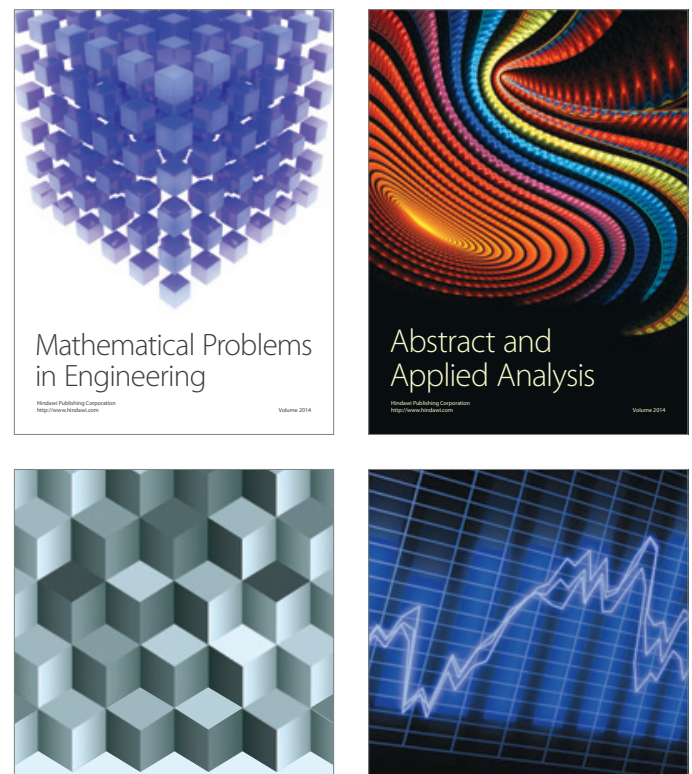

Journal of

Function Spaces

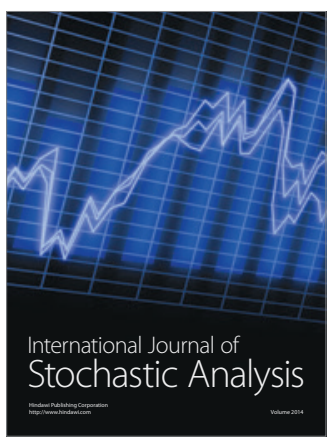

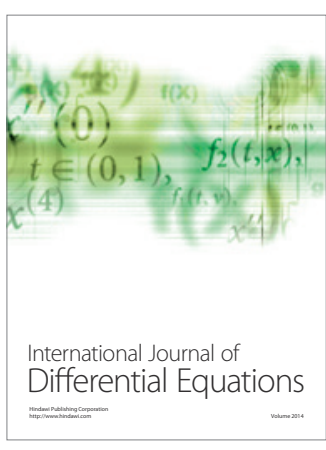
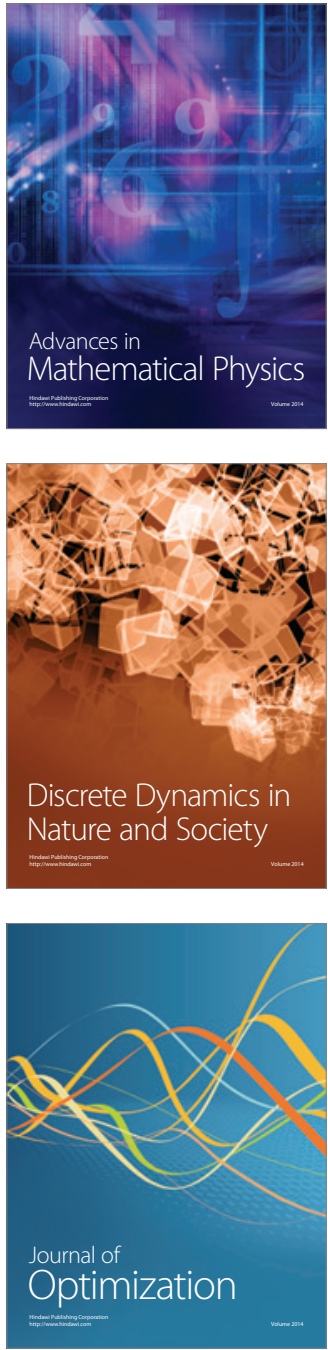